\title{
Índice de qualidade do solo em cultivo agroecológico e convencional no semiárido potiguar, Brasil
}

\section{Index of soil quality in agroecological and conventional farming in potiguar semiarid, Brazil}

\author{
Fábio dos Santos Santiago ${ }^{1}$, Suzana Maria Gico Lima Montenegro ${ }^{2}$, Maria Rafaela de Almeida Pinheiro ${ }^{3}$
}

Resumo: O uso e manejo inadequado em sistemas de cultivo na região semiárida podem declinar a capacidade do solo em sustentar a produção. O objetivo do estudo foi determinar o índice de qualidade do solo (IQS) em unidades experimentais agrícolas (UEA) irrigadas agroecológica e convencional, localizadas na comunidade de Sombras Grandes, Caraúbas, Rio Grande do Norte. As amostras simples de solo $(0-20 \mathrm{~cm})$ foram coletadas no período de 2009 a 2012. Para determinar o IQS, utilizaram-se 11 indicadores de qualidade associados às funções do solo em manter a capacidade produtiva ao longo do tempo: densidade do solo (Ds), porosidade total (PT), resistência à penetração (RP), disponibilidade de água no solo (AD/PT), retenção de umidade a $-33 \mathrm{kPa}$ (UVcc/PT), $\mathrm{pH}$, fósforo assimilável $(\mathrm{P})$, capacidade de troca catiônica (CTC), saturação de bases (V), matéria orgânica do solo (MOS), argila dispersa em água (ADA) e grau de floculação (GF). O IQS nas UEAs agroecológica e convencional foram classificados como ótimo $(0,80)$ e ruim $(0,45)$, respectivamente. Na UEA convencional há necessidade de práticas agrícolas como aporte de resíduos orgânicos que melhorem o GF, pois foi o indicador que mais contribuiu para reduzir o IQS. O IQS reflete a integração dos atributos químicos e físicos, podendo ser utilizado nas tomadas de decisão sobre o uso e manejo do solo.

Palavras-chave: Indicadores físicos; Indicadores químicos; Agroecologia; Agricultura familiar.

\begin{abstract}
The inadequate use and management in cropping systems in the semiarid region may decline the soil's ability to sustain the production. The objective of this work was to determine the soil quality index (SQI) in agroecological and conventional irrigated agricultural experimental units (AEU), located in the community of Sombras Grandes, Caraúbas, Rio Grande do Norte. Simple soil samples $(0-20 \mathrm{~cm})$ were collected from 2009 to 2012. To determine the SQI, it was used 11 quality indicators associated to the soil functions in maintain the productivity capacity over time: bulk density (Bd), total porosity (TP), penetration resistance (PR), water availability in soil (AWS/TP), moisture retention at $-33 \mathrm{kPa}(\mathrm{MR} / \mathrm{TP})$, $\mathrm{pH}$, phosphorus assimilable (P), cation exchange capacity (CEC), base saturation (V), soil organic matter (SOM), clay dispersed in water (CDW) and flocculation degree (FD). The SQI in agroecological and conventional irrigated AEU were classified as good (0.80) and bad (0.45), respectively. In conventional AEU there is need for agricultural practices, like injection of organic waste, that improve the FD because it was the indicator that most contributed to reduce the SQI. The SQI reflected the integrated performance of the chemical and physical properties, which can be used in making decisions about the soil use and management.
\end{abstract}

Key words: Physical indicators; Chemical indicators; Agroecology; Family farming.

\footnotetext{
*Autor para correspondência

Recebido para publicação em 11/11/2017; aprovado em 20/12/2017

${ }^{1}$ Doutor em Engenharia Agrícola pela Universidade Federal Rural de Pernambuco, Coordenador Técnico do Projeto Dom Helder Câmara-Ministério do Desenvolvimento Agrário /Fundo Internacional para Desenvolvimento da Agricultura. Rua Doutor Silva Ferreira, 122 , Santo Amaro, CEP 50040130 - Recife, (81)996011716, fabioirriga@ hotmail.com

${ }^{2} \mathrm{PhD}$ em Engenharia Civil pela University of Newcastle Upon Tyne, Professora do Departamento de Engenharia Civil e Recursos Hídricos da Universidade Federal de Pernambuco, suzanam.ufpe@ gmail.com

${ }^{3}$ Engenheira Agrícola e Ambiental pela Universidade Federal Rural de Pernambuco, Monitora Ambiental do Projeto Dom Helder Câmara-Ministério do Desenvolvimento Agrário/Fundo Internacional para Desenvolvimento da Agricultura, m.rafaelapinheiro@yahoo.com.br
} 


\section{INTRODUÇÃO}

O Semiárido do Nordeste do Brasil é caracterizado por déficit hídrico, apresenta normalmente baixa matéria orgânica do solo devido a processos de erosão, elevadas temperaturas e reduzida reposição de resíduos vegetais. O uso e manejo inadequado em sistema de cultivo irrigado na região semiárida podem declinar a capacidade do solo em sustentar a produção em tempo reduzido (SANTIAGO, 2015).

Os solos na região semiárida são susceptíveis à degradação, como as áreas de produção familiar irrigadas, devido aos processos de salinização e/ou sodicidade, erosão, compactação, monocultivo, lixiviação de nutrientes e aplicação continuada de adubos mineralizados. Essa dinâmica proporciona uma agricultura itinerante à busca de novas áreas sobre a caatinga, gerando a perda da biodiversidade do ecossistema e a vulnerabilidade da produção de alimentos na região (SANTIAGO, 2015).

Neste cenário, é fundamental que o uso e manejo do solo em sistemas agrícolas na região semiárida possam manter sua capacidade produtiva de colheitas de alimentos e/ou fibras ao longo do tempo. Para tanto, deve-se estabelecer como principais funções do solo a partir das práticas agrícolas: crescimento radicular (CR), condução e armazenamento de água (CAA), suprir e ciclar nutrientes $(\mathrm{SCN})$ e resistir à degradação (RD) (CÂNDIDO, 2014).

Os indicadores físicos e químicos têm sido amplamente utilizados para a determinação de índices de qualidade do solo (BHARDWAJ et al., 2011).

Os índices de qualidade do solo são ferramentas que se destinam a tornar a informação complexa sobre a saúde do solo ou a qualidade mais acessível e interpretável para os tomadores de decisão (MCBRATNEY et al., 2013). A seleção de indicadores de qualidade do solo e a integração destes com as funções do solo em um único índice poderá fornecer informações sobre a mudança da qualidade do solo e, em seguida, orientar as decisões sobre o uso e manejo dos sistemas de cultivo (CHEN et al., 2013).

Entre os índices mais utilizados para avaliação da qualidade do solo, destaca-se o índice de qualidade do solo (IQS) proposto por Karlen e Stott (1994). O IQS apresenta um modelo aditivo em que as funções principais e seus respectivos indicadores são selecionados e ponderados de acordo com o grau de importância. Aqueles pesquisadores sugeriram a utilização de um modelo desenvolvido por Wymore (1993) para normalização dos indicadores, gerando uma escala de 0 a 1 , significando que, quanto mais próximo de 1 , maior a qualidade do solo vinculada ao aspecto em análise.

Existem poucos trabalhos científicos que possam comparar índice de qualidade do solo entre sistemas de cultivo irrigado em área de agricultura familiar no Semiárido nordestino. O presente estudo teve como objetivo determinar o índice de qualidade do solo (IQS) em unidades experimentais agrícolas (UEAs) agroecológica e convencional.

\section{MATERIAL E MÉTODOS}

O experimento foi constituído em duas UEAs. Sendo uma agroecológica e a outra convencional. As UEAs estão localizadas na mesma comunidade de agricultores familiares de Sombras Grandes, Caraúbas/RN, mesorregião Oeste Potiguar e microrregião Chapada do Apodi, latitude de $5^{\circ}$
37'7548', S e longitude de $37^{\circ} 26^{\prime} 43^{\prime}$ ' W. Os solos nas áreas estudadas são originados do embasamento sedimentar calcário e classificados como Cambissolo eutrófico (EMBRAPA, 2006).

A região possui clima semiárido do tipo BShw', segundo Köppen, megatérmico, precipitação média anual de 400 a $600 \mathrm{~mm}$, com períodos chuvosos variando de janeiro a julho e secos de julho a dezembro. Os meses de maiores precipitações são de março a abril. A vegetação predominante é do tipo Caatinga Hiperxerófila. Temperatura média máxima é de $37{ }^{\circ} \mathrm{C}$ e mínima de $21{ }^{\circ} \mathrm{C}$, média anual de $26{ }^{\circ} \mathrm{C}$ com amplitude de $7^{\circ} \mathrm{C}$ (LIRA et al., 2012).

$\mathrm{O}$ registro sobre o histórico das áreas segue a proposta realizada por Obade e Lal (2014), em estudo sobre sustentabilidade de cinco sistemas de cultivo no Estado de Ohio, EUA, que descreveram as principais intervenções no manejo dos sistemas agrícolas para associar índices de qualidade do solo com a sustentabilidade ambiental.

Neste sentido, segue o histórico das UEAs onde foram irrigadas com água $\mathrm{C}_{3} \mathrm{~S}_{1}$, ou seja, água de salinidade alta e alcalinidade baixa (RICHARDS, 1954). A UEA agroecológica até 1998 era recoberta por vegetação de caatinga natural. Em 1999, iniciou-se a retirada da vegetação e o preparo do solo foi realizado através de trator com grade mecânica para os cultivos de sequeiro de milho (Zea mays L.) e feijão de corda (Vigna unguiculata (L.) Walp.) que se prolongou até 2001. Neste período, não foram usados adubos mineralizado e orgânico, nem queimada dos resíduos vegetais.

A UEA agroecológica ficou em pousio até meados de 2003. Foi retomado o plantio de sequeiro entre 2004 a 2005 com retirada das espécies arbóreas remanescentes, roço e uso de trator com grade mecânica. Em meados de 2006 foi iniciada a produção irrigada agroecológica de hortaliças e frutas em 0,8 ha, com a utilização do sistema de irrigação por microaspersão. Utilizaram-se as seguintes práticas agroecológicas: preparo da terra com arado de aiveca/tração animal e enxada nos canteiros; aplicação de $10 \mathrm{t}$ composto ha ${ }^{1}$ ano $^{-1}$ e $4 \mathrm{t}$ de esterco bovino ha ${ }^{-1}$ ano $^{-1}$; cobertura morta (palhadas de carnaúba e capim elefante); controle de pragas e doenças com biofertilizante (5\%) e urina de vaca (1\%); rotação de culturas; pousio e adubação verde; e diversidade de cultivo.

$\mathrm{Na}$ UEA convencional em 1995, a vegetação predominante era de caatinga em fase de recuperação. Em 1996, a UEA convencional foi desmatada com uso da queimada e preparo do solo com arado e grade de arrasto para o cultivo de hortaliças de base convencional até 2003 e ficou em pousio até 2006 . Em 2007, foi retomada a produção de hortaliças irrigadas numa área de 1,0 ha através de sistema de irrigação por aspersão. $\mathrm{O}$ uso do trator com grade mecânica foi utilizado anualmente para revolvimento do solo e confecção dos canteiros. Houve aplicação anual de adubo mineralizado com $180 \mathrm{Kg}_{\text {de }} \mathrm{N} \mathrm{ha}^{-1}, 120 \mathrm{~kg}$ de $\mathrm{K}_{2} \mathrm{O}^{-1}{ }^{-1}, 100$ $\mathrm{kg}$ de $\mathrm{P}_{2} \mathrm{O}_{5} \mathrm{ha}^{-1}$, utilizando como fontes ureia, cloreto de potássio e superfosfato simples, respectivamente. Anualmente aplicaram-se em média 2 toneladas de esterco bovino. No controle de pragas e doenças foram utilizados agrotóxicos, com predominância de Deltametrina (Decis) e Folidol.

Foram selecionados indicadores físicos e químicos, tendo como referência os utilizados por Miranda (2012) para a indexação da qualidade do solo, tais como: densidade do solo (Ds), porosidade total (PT), resistência à penetração 
(RP), relação entre água disponível pela porosidade total $(\mathrm{AD} / \mathrm{PT})$, relação entre a umidade volumétrica na capacidade de campo pela porosidade total (UVcc/PT), $\mathrm{pH}$, fósforo assimilável (P), capacidade de troca catiônica (CTC), saturação de bases (V), matéria orgânica do solo (MOS) e grau de floculação (GF).

Os indicadores de qualidade para composição do índice foram selecionados mediante a meta do manejo em manter a capacidade produtiva do solo, associado às funções de sustentabilidade ambiental na região semiárida como a $\mathrm{CR}$, CAA, SCN e RD.

Para tanto, foram coletadas amostras simples de solo (deformadas e indeformadas) nas UEAs durante o período de 2009 a 2012. Foi utilizada uma malha amostral para cada área de 0,5 ha, definida em transecto com 5 pontos equidistantes entre si $(10 \mathrm{~m})$. Em cada UEA foram coletadas 20 amostras de solo, sendo 5 a cada ano, na camada de 0-20 cm, totalizando 40 amostras.

As análises físicas incluíram granulometria e argila dispersa em água (ADA) pelo método do densímetro; a densidade do solo (Ds) pelo método do Anel volumétrico (amostra indeformada); a densidade das partículas (Dp) pelo balão volumétrico, a porosidade total $\left(P T=1-\frac{D s}{D p}\right)$.

$\mathrm{O}$ grau de floculação $(\mathrm{GF})$ foi determinado em função da argila total e ADA. A umidade volumétrica na capacidade de campo (CC) a um potencial mátrico de $-33 \mathrm{kPa}$ e ponto de murcha permanente (PMP) a $-1.500 \mathrm{kPa}$, utilizando a câmara de Richards com placas porosas (amostra indeformada). A água disponível foi calculada pela diferença entre a $\mathrm{CC}$ e PMP (EMBRAPA, 2009).

A resistência mecânica do solo à penetração (RP) foi avaliada com a utilização de penetrômetro de impacto modelo IAA/Planalsucar - Stolf, até profundidade de $0,20 \mathrm{~m}$, intervalos de $0,05 \mathrm{~m}$, em 5 pontos em cada área com 5 repetições, totalizando 75 medições intervalo ${ }^{-1}$ (STOLF, 1991). As médias desses valores foram multiplicadas por 0,098 para obtenção da RP em MPa e obtida uma média geral para a camada de $0-20 \mathrm{~cm}$.

As análises químicas foram realizadas em amostras simples de solo deformadas. $\mathrm{O} \mathrm{pH}$ foi medido em água (1:2,5); o P disponível foi extraído por Mehlich-1 e dosado por calorimetria (EMBRAPA, 2006; BRAGA; DEFELIPO, 1974); o carbono orgânico total (COT) foi determinado pelo método Walkley e Black (1934), descrito por Mendonça e Matos (2005); os cátions trocáveis $\mathrm{Ca}^{+2}, \mathrm{Mg}^{+2}, \mathrm{~K}^{+}$e $\mathrm{Na}^{+}$ foram extraídos com solução de acetato de amônio $\left(1 \mathrm{~mol} \mathrm{~L}^{-1}\right)$

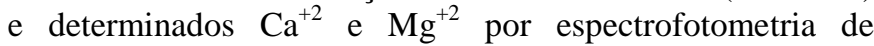
absorção atômica e $\mathrm{K}^{+}$e $\mathrm{Na}^{+}$por fotometria de emissão de chama; a acidez potencial $(\mathrm{H}+\mathrm{Al})$ foi determinada conforme a Embrapa (2009). Com os resultados dos indicadores químicos foram calculadas a soma de bases (SB), capacidade de troca de cátions (CTC), saturação por bases (V \%) e a matéria orgânica do solo (MOS) (EMBRAPA, 2009).

$\mathrm{Na}$ proposta de cálculo do IQS é realizada a normalização dos valores dos indicadores em escala única, ou seja, entre 0 e 1 . Os indicadores não apresentam unidades similares. A ferramenta para obtenção do escore normalizado do indicador é a função para padronização, desenvolvido para sistemas de engenharia por Wymore em 1993 (YAO et al., 2013), como pode ser visto na Equação 1.

$$
v=\frac{1}{1+\left(\frac{B-L}{x-L}\right)^{2 S(B+x-2 L)}}
$$

Em que: $v$ é a pontuação padronizada do indicador; $\mathrm{B}$, o valor crítico ou limite base do indicador, cujo escore padronizado é 0,5 e que coloca o limite entre a ruim e a boa qualidade do solo; L, o valor inicial ou mais baixo que o atributo pode expressar, podendo ser igual a $0 ; \mathrm{S}$, a inclinação da tangente à curva no ponto associado ao valor crítico do indicador; e x, o valor do indicador medido no campo.

$\mathrm{Na}$ aplicação da equação de padronização de escores dos indicadores é necessário, calcular a inclinação (S) da tangente da curva de pontuação no valor crítico do indicador. Derivando $S$ na Equação 1, tem-se a Equação 2.

$$
S=\frac{\log \left(\frac{1}{v}\right)-1}{\log \left(\frac{B-L}{x-L}\right) \cdot 2(B+x-2 L)}
$$

Na padronização dos indicadores foram utilizados dois valores limite (superior e inferior) e um valor crítico (limite base). Os valores-limite superiores correspondem os dos indicadores de qualidade em que a função de pontuação equivale a 1, quando o atributo está em nível ótimo. Os valores-limite inferiores são aqueles dos indicadores de qualidade onde a função de pontuação equivale a zero. Os valores críticos são aqueles em que a função de pontuação é igual a 0,5 .

No cálculo de $\mathrm{S}$ deve-se utilizar o valor $v$ igual a 0,5 . Foram geradas três funções típicas de padronização dos dados (Figura 1): (a) "mais é melhor"; (b) "valor máximo (ótimo)"; (c) "menos é melhor" (WYMORE, 1993).

Figura 1. Funções de pontuação padronizada: (a) "mais é melhor”; (b) "valor máximo (ótimo); (c) "menos é melhor".
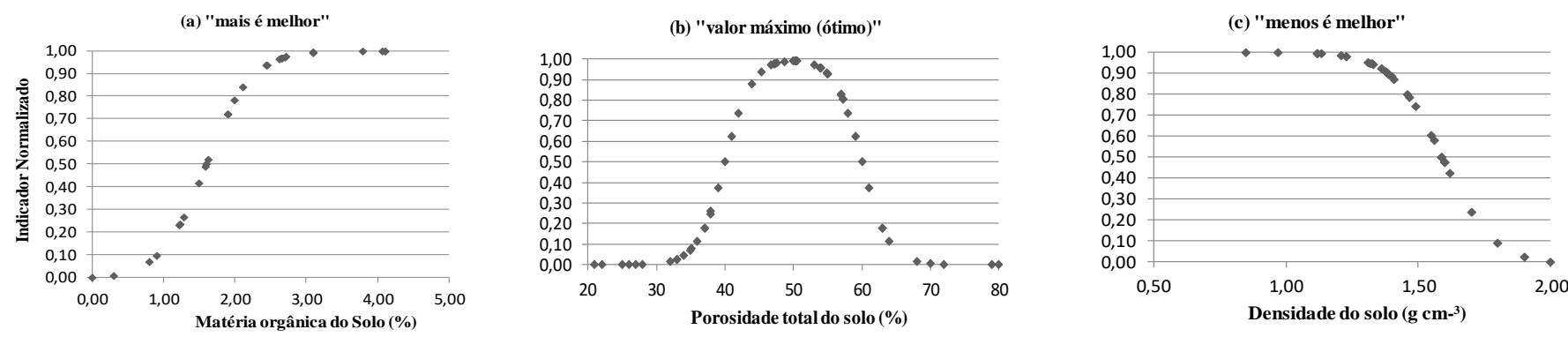

A curva de padronização do tipo "mais é melhor" possui inclinação da tangente (S) positiva e foi utilizada para normalizar indicadores em que os maiores valores melhoram a qualidade do solo, como exemplo a V, CTC, UVcc/PT, P, MOS, GF, AD/PT. A do tipo "máximo (ótimo)" possui $\mathrm{S}$ positiva até o valor máximo e foi usada para indicadores

como pH, PT que apresentam comportamento de melhorar a qualidade do solo até determinado valor, e a partir deste sua influência é negativa. A curva "menos é melhor" possui $\mathrm{S}$ negativa e é direcionada para indicadores que apresentam quanto menor valor é melhor para a qualidade do solo, como a Ds e RP. 
De modo a "quantificar" a qualidade do solo, foi considerada a estrutura de cálculo do IQS mediante a Tabela 1. O modelo foi proposto por Karlen e Stott (1994) e adaptado por Melo Filho et al. (2009), Silva et al. (2013), Monteiro (2012), Miranda (2012), Alvarenga et al. (2012).

Tabela 1. Funções principais, indicadores físicos e químicos, valores-limite (inferior, ótimo e superior) e base (crítico) e inclinação da tangente (S), bem como os pesos dos indicadores a partir do grau a serem utilizados na avaliação da qualidade do solo nas UEAs A e C.

\begin{tabular}{|c|c|c|c|c|c|c|c|c|c|c|c|}
\hline Função principal & $\begin{array}{l}\text { Ponderador } \\
\text { da função }\end{array}$ & $\begin{array}{c}\text { Indicador } \\
\text { de } \\
\text { qualidade }\end{array}$ & $\begin{array}{l}\text { Ponderador } \\
\text { do indicador }\end{array}$ & $\begin{array}{l}\text { Tipo da } \\
\text { curva }\end{array}$ & $\begin{array}{l}\text { Limite } \\
\text { inferior }\end{array}$ & $\begin{array}{l}\text { Limite } \\
\text { base } \\
\text { inferior }\end{array}$ & Ótimo & $\begin{array}{c}\text { Limite } \\
\text { base } \\
\text { superior }\end{array}$ & $\begin{array}{l}\text { Limite } \\
\text { superior }\end{array}$ & $\begin{array}{l}\text { Referências } \\
\text { dos limites }\end{array}$ & $\begin{array}{l}\text { Inclinação } \\
\text { da tangente } \\
\text { (S) }\end{array}$ \\
\hline \multirow{4}{*}{$\begin{array}{c}\text { Crescimento } \\
\text { Radicular (CR) }\end{array}$} & \multirow{4}{*}{0,30} & Ds $\left(\mathrm{g} \mathrm{cm}^{-3}\right)$ & 0,20 & $\begin{array}{l}\text { "Menos é } \\
\text { melhor" }\end{array}$ & 0,85 & 1,59 & - & 2 & - & $\begin{array}{l}\text { Chaer } \\
\text { (2001) }\end{array}$ & $-2,6170$ \\
\hline & & RP (Mpa) & 0,30 & $\begin{array}{l}\text { "Menos é } \\
\text { melhor" }\end{array}$ & - & - & - & 2 & 4 & $\begin{array}{l}\text { Miranda } \\
(2012)\end{array}$ & $-0,4167$ \\
\hline & & PT (\%) & 0,20 & "Ótimo" & 20 & 40 & 50 & 60 & 80 & $\begin{array}{l}\text { Glover et al. } \\
\text { (2001) }\end{array}$ & 0,1280 \\
\hline & & $\operatorname{MOS}(\%)$ & 0,30 & $\begin{array}{l}\text { "Mais é } \\
\text { melhor" }\end{array}$ & 0 & 1,6 & - & - & 3,2 & $\begin{array}{c}\text { Glover et al. } \\
\text { (2000) }\end{array}$ & $-2,6200$ \\
\hline \multirow{4}{*}{$\begin{array}{c}\text { Condução e } \\
\text { Armazenamento } \\
\text { de Água (CAA) }\end{array}$} & \multirow{4}{*}{0,25} & Ds $\left(\mathrm{g} \mathrm{cm}^{-3}\right)$ & 0,20 & $\begin{array}{l}\text { "Menos é } \\
\text { melhor" }\end{array}$ & 0,85 & 1,59 & - & 2 & - & $\begin{array}{l}\text { Chaer } \\
\text { (2001) }\end{array}$ & $-2,6170$ \\
\hline & & $\operatorname{MOS}(\%)$ & 0,20 & $\begin{array}{l}\text { "Mais é } \\
\text { melhor" }\end{array}$ & 0 & 1,6 & - & - & 3,2 & $\begin{array}{l}\text { Glover et al. } \\
\quad(2000)\end{array}$ & $-2,6200$ \\
\hline & & $\mathrm{Uv}_{\mathrm{cd}} / \mathrm{PT}$ & 0,30 & $\begin{array}{l}\text { "Mais é } \\
\text { melhor" }\end{array}$ & 0 & 0,55 & - & - & - & $\begin{array}{l}\text { Souza } \\
(2005)\end{array}$ & 5,005 \\
\hline & & $\mathrm{AD} / \mathrm{PT}$ & 0,30 & $\begin{array}{l}\text { "Mais é } \\
\text { melhor" }\end{array}$ & 0 & 0,125 & - & - & - & $\begin{array}{l}\text { Souza } \\
(2005)\end{array}$ & 0,125 \\
\hline \multirow{5}{*}{$\begin{array}{c}\text { Suprir e ciclar } \\
\text { nutrientes }(\mathrm{SCN})\end{array}$} & \multirow{5}{*}{0,30} & $\mathrm{pH}$ & 0,10 & "Ótimo" & 5 & 5,3 & 7 & 8,4 & 9 & $\begin{array}{c}\text { Fernandes } \\
(2008)\end{array}$ & 0,5005 \\
\hline & & $\begin{array}{c}\text { CTC } \\
\left(\mathrm{cmolc} \mathrm{kg}^{-1}\right)\end{array}$ & 0,30 & $\begin{array}{l}\text { "Mais é } \\
\text { melhor" }\end{array}$ & 0 & 6,5 & - & - & 18 & $\begin{array}{c}\text { Ribeiro, } \\
\text { Gontijo e } \\
\text { Alvarez V. } \\
\text { (1999) }\end{array}$ & 0,1282 \\
\hline & & $\mathrm{V}(\%)$ & 0,20 & $\begin{array}{l}\text { "Mais é } \\
\text { melhor" }\end{array}$ & 0 & 50 & - & - & 100 & $\begin{array}{c}\text { Ribeiro, } \\
\text { Gontijo e } \\
\text { Alvarez V. } \\
\text { (1999) }\end{array}$ & 0,0250 \\
\hline & & $\mathrm{P}\left(\mathrm{mg} \mathrm{kg}^{-1}\right)$ & 0,10 & $\begin{array}{l}\text { "Mais é } \\
\text { melhor" }\end{array}$ & 0 & 10 & - & - & & $\begin{array}{l}\text { Cinira } \\
(2008)\end{array}$ & 0,1251 \\
\hline & & $\operatorname{MOS}(\%)$ & 0,30 & $\begin{array}{l}\text { "Mais é } \\
\text { melhor" }\end{array}$ & 0 & 1,6 & - & - & 3,2 & $\begin{array}{c}\text { Glover et al. } \\
\text { (2000) }\end{array}$ & $-2,6200$ \\
\hline \multirow{2}{*}{$\begin{array}{c}\text { Resistir à } \\
\text { Degradação (RD) }\end{array}$} & \multirow{2}{*}{0,25} & GF $(\%)$ & 0,4 & $\begin{array}{l}\text { "Menos é } \\
\text { melhor" }\end{array}$ & 0 & 50 & - & - & - & $\begin{array}{l}\text { Goedert } \\
\text { (2005) }\end{array}$ & 0,0600 \\
\hline & & $\operatorname{MOS}(\%)$ & 0,6 & $\begin{array}{l}\text { "Mais é } \\
\text { melhor" }\end{array}$ & 0 & 1,6 & - & - & 3,2 & $\begin{array}{c}\text { Glover et al. } \\
(2000)\end{array}$ & $-2,6200$ \\
\hline
\end{tabular}

Ds: densidade do solo; RP: resistência à penetração; PT: porosidade total; MOS: matéria orgânica do solo; UVcc/PT: relação da umidade volumétrica na capacidade de campo e a porosidade total; AD/PT: relação da água disponível e a porosidade total; CTC: capacidade de troca catiônica; V: saturação de bases; P: fósforo; GF: grau de floculação

As funções do solo e os respectivos pesos foram estabelecidos na Tabela 1, bem como os pesos dos indicadores a partir do grau de importância que exerce em cada função a meta estabelecida para o manejo, adaptados os propostos por Miranda (2012). Este método consiste em um sistema aditivo e utiliza uma série de funções do solo, nas quais são atribuídos pesos e são integradas de acordo com a Equação 3.

$$
I Q S=\sum \mathrm{qWi}(\mathrm{wt})
$$

Em que: IQS é o índice de qualidade do solo; qWi, o valor calculado para as funções principais que fazem parte do índice; e wt, um peso numérico atribuído a cada função na composição geral de qualidade.

O somatório dos pesos relacionados às funções definidas deve ser necessariamente igual a 1,0. Este é o valor do IQS para um solo considerado de qualidade mediante o objetivo da avaliação. Por outro lado, quando o solo apresenta limitações dos seus indicadores de qualidade o valor vai se aproximando de zero. Para tanto, foram definidas quatro funções principais do solo, seus respectivos pesos e indicadores de qualidade (Tabela 1): crescimento radicular (CR); condução e armazenamento de água (CAA); suprir e ciclar nutrientes $(\mathrm{SCN})$; resistir à degradação (RD). Assim, o cálculo se deu por meio da Equação. 4:

$$
q(F P)=I_{1} \times W_{1}+\cdots+I_{n} \times W_{n}
$$

Em que $q(F P)$ é a contribuição parcial de cada função principal para a determinação do valor global do IQS; $I_{n}$ os valores dos indicadores da função principal (FP) avaliada; e Wn os pesos relativos atribuídos a cada indicador.

Para o cálculo do IQS, foi considerado o valor médio do indicador. Cada indicador teve um escore padronizado de acordo com as funções de padronização. Assim, os valores de $\mathrm{I}_{\mathrm{n}}$ compreenderam entre 0 e 1 .

$\mathrm{Na}$ etapa seguinte, foram multiplicados os valores encontrados em cada FP pelo seu respectivo ponderador e efetuada a soma dos resultados. O IQS foi determinado por meio da Equação 5.

$$
I Q S=(q C R \times w C R)+(q C A A x w C A A)+
$$

$(q S C N \times w S C N)+(q R D \times w R D)$

Em que: qCR - valor ponderado da FP crescimento radicular; qCAA - FP da capacidade de armazenamento de água; qSCN - FP de suprir e ciclar nutrientes; qRD - FP de 
resistir à degradação; w - ponderadores associados a cada função principal.

\section{RESULTADOS E DISCUSSÃO}

O Cambissolo eutrófico nas UEA agroecológica e convencional apresentam classe textural franco-arenosa na camada estudada $(0-20 \mathrm{~cm})$ (Tabela 2). Isso é importante, haja vista que práticas de uso e manejo em solos de textura diferentes resultam em comportamentos diferentes aos indicadores de qualidade do solo (CORRÊA et al., 2009). A UEA agroecológica apresentou valor menor de Ds $\left(1,32 \mathrm{~g} \mathrm{~cm}^{-}\right.$ ${ }^{3}$ ) em relação à UEA convencional $\left(1,60 \mathrm{~g} \mathrm{~cm}^{-3}\right)$ (Tabela 3$)$. De acordo com Kiehl (1979), a Ds deve permanecer no intervalo entre 1,1 a $1,6 \mathrm{~g} \mathrm{~cm}^{-3}$ em solos minerais, assumindo valores a $1,6 \mathrm{~g} \mathrm{~cm}^{-3} \mathrm{em}$ solos de textura arenosa. Camargo e Alleoni (1997) assinalaram como crítico, em solos variando de franco-argilosos a argilosos, o valor da Ds na ordem de $1,55 \mathrm{~g} \mathrm{~cm}^{-3}$ já que apresenta problemas de enraizamento para as culturas, indicando a necessidade de adoção de práticas agrícolas para redução da Ds.

A PT na UEA agroecológica foi $50,02 \%$ e na UEA convencional de 37,95\% (Tabela 3). A UEA agroecológica apresentou valor de PT no limite mínimo estabelecido por Kiehl (1979) e Azevedo e Dalmolin (2006), que é de 50\%. Na UEA agroecológica foi encontrado valor menor que $2 \mathrm{MPa}$ para RP (1,41 MPa). Já na UEA convencional, o valor de RP (2,21 MPa) passou do limite de $2 \mathrm{MPa}$ em 10,5\% (Tabela 3). Valores críticos de resistência à penetração podem variar de 1,5 a $4 \mathrm{MPa}$ (ROSOLEM et al., 1999); no entanto, valores próximos a $2 \mathrm{MPa}$ são, de maneira geral, aceitos como impeditivos ao crescimento radicular (BLAINSKI et al., 2008).

Tabela 2. Composição granulométrica dos solos nas unidades experimentais agrícolas agroecológica e convencional (0-20 $\mathrm{cm})$.

\begin{tabular}{ccc}
\hline \multirow{2}{*}{ Indicador } & \multicolumn{2}{c}{ Sistemas de cultivo } \\
\cline { 2 - 3 } & UEA agroecológica & UEA convencional \\
\cline { 2 - 3 } & Média & Média \\
\hline Areia Grossa (\%) & 54,74 & 30,27 \\
\hline Areia Fina (\%) & 26,34 & 39,43 \\
\hline Silte (\%) & 6,58 & 19,22 \\
\hline Argila (\%) & 12,34 & 11,07 \\
\hline UEA: unidade experimental agrícola.
\end{tabular}

Foi observado que o valor menor de RP na UEA agroecológica está associado com o menor resultado verificado de $\mathrm{ADA}$ e Ds, assim como maior PT em relação à UEA convencional. Por outro lado, na UEA agroecológica o valor do GF foi maior que a UEA convencional. De acordo com Mota et al. (2013), a presença de ADA pode contribuir para o aumento da RP. Além disso, o baixo GF contribui para pior estruturação do solo e consequentemente aumento da RP, devido ao aumento da ADA.

A relação UVcc/PT foi maior na UEA convencional $(0,44)$ em relação à UEA agroecológica $(0,23)$ (Tabela 3$)$. No entanto, ambas as UEAs apresentaram resultados menores ao limite proposto de 0,55 por Souza (2005). Na UEA agroecológica, nota-se que apenas $23 \%$ da porosidade total é constituída de UVcc e associada ao teor de umidade com baixa tensão da matriz do solo (-33 kPa); e ao mesmo tempo, verifica-se que o teor de areia total foi $81,08 \%$, PT de $50,02 \%$ e Ds igual $1,32 \mathrm{~g} \mathrm{~cm}^{-3}$ que provavelmente predominaram macroporos, considerados de menor capacidade de retenção de água do solo e maior percolação.

$\mathrm{Na}$ UEA convencional observa-se o valor para UVcc/PT de 0,44 , ainda abaixo do crítico $(0,55)$, mesmo assim apresentou melhores condições que a UEA agroecológica $(0,23)$ (Tabela 3$)$, provavelmente houve predominância de poros de maior retenção de água no solo (microporos). Isso se associa a maior valor de Ds $\left(1,60 \mathrm{~g} \mathrm{~cm}^{-3}\right)$ e menor valor de PT $(37,02 \%)$. Pode-se associar essas condições com a passagem continuada de máquinas agrícolas, ausência de matéria orgânica e menor aporte de resíduos no solo. Este comportamento se aproxima aos encontrados por Melo Filho et al. (2009) e Monteiro (2012) que obtiveram Ds próximo a $1,60 \mathrm{~g} \mathrm{~cm}^{-3}$ com maior relação de UVcc/PT.

O limite base (crítico) de AD/PT proposto é de 0,125 (SOUZA, 2005). Na UEA agroecológica, foi observado o valor 0,11 , ou seja, $11 \%$ da AD compõe a PT, onde o mínimo recomendado seria de $12,5 \%$. Na UEA convencional, o valor foi de 0,22 , representando $76 \%$ a mais do valor crítico. De forma geral, a UEA convencional apresentou melhores valores de UVcc/PT e $\mathrm{AD} / \mathrm{PT}$ em relação à UEA agroecológica (Tabela 3). Neste sentido, indicadores com PT e Ds não devem ser avaliados de forma separada e sim em função com outros indicadores inter-relacionados como a retenção e capacidade de armazenamento de água no solo.

Tabela 3. Valores médios de indicadores químicos e físicos nas unidades experimentais agrícolas agroecológica e convencional $(0-20 \mathrm{~cm})$.

\begin{tabular}{|c|c|c|c|c|c|c|c|c|c|c|c|c|c|c|c|c|c|c|c|}
\hline $\begin{array}{c}\text { Sistema } \\
\text { de } \\
\text { Cultivo }\end{array}$ & $\mathrm{pH}$ & $\mathrm{Ca}$ & $\mathrm{Mg}$ & $\mathrm{K}$ & $\begin{array}{c}\mathrm{Na} \\
\mathrm{ol}_{(+)} / \mathrm{Kg}\end{array}$ & $\mathrm{Al}+\mathrm{H}$ & CTC & SB & $\begin{array}{r}\mathrm{V} \\
(\%) \\
\end{array}$ & $\begin{array}{c}\text { MOS } \\
(\%)\end{array}$ & $\begin{array}{c}\mathrm{P} \\
(\mathrm{mg} / \mathrm{Kg})\end{array}$ & $\begin{array}{c}\text { Ds } \\
\left(\mathrm{g} \mathrm{cm}^{3}\right)\end{array}$ & $\begin{array}{c}\mathrm{RP} \\
(\mathrm{MPa})\end{array}$ & PT & ADA & GF & $\mathrm{AD}$ & $\begin{array}{l}\mathrm{AD} / \\
\mathrm{PT}\end{array}$ & $\begin{array}{c}\text { UVcc } \\
\text { /PT }\end{array}$ \\
\hline $\begin{array}{c}\text { UEA } \\
\text { Agroec } \\
\text { ológica }\end{array}$ & 7,79 & 8,67 & 3,69 & 0,57 & 0,73 & 1,93 & 15,59 & 13,66 & 85,62 & 2,44 & 111,23 & 1,32 & 1,41 & 50,02 & 5,58 & 54,73 & 5,16 & 0,11 & 0,23 \\
\hline $\begin{array}{c}\text { UEA } \\
\text { Conven } \\
\text { cional }\end{array}$ & 7,09 & 7,74 & 3,31 & 1,10 & 0,70 & 2,21 & 15,06 & 12,85 & 84,00 & 1,22 & 160,55 & 1,60 & 2,21 & 37,95 & 7,99 & 25,99 & 8,20 & 0,22 & 0,44 \\
\hline
\end{tabular}

UEA: unidade experimental agrícola; pH: potencial hidrogeniônico; Ca: cálcio; Mg: magnésio; K: potássio; Na: sódio; Al+H: alumínio e hidrogênio; CTC: capacidade de troca catiônica; SB: soma de bases; V: saturação de bases; MO: matéria orgânica; P: fósforo; Ds: densidade do solo; PT: porosidade total; ADA: argila dispersa em água; GF: grau de floculação; AD: Água disponível; AD/PT: relação entre água disponível e porosidade total; UVcc/PT: relação entre a umidade na capacidade de campo e a porosidade total.

Notadamente, os valores de $\mathrm{pH}$ encontrados nos tratamentos (UEA agroecológica e UEA convencional) estão em consonância como altos teores de cátions básicos $\left(\mathrm{Ca}^{+2} \mathrm{e}\right.$ $\left.\mathrm{Mg}^{+2}\right)$ e baixos teores de acidez potencial $(\mathrm{H}+\mathrm{Al})$. Dantas et al. (2012) verificaram que a adição de restos vegetais ao solo promoveu neutralização da acidez potencial, aumentando as cargas negativas do solo disponíveis para a adsorção de

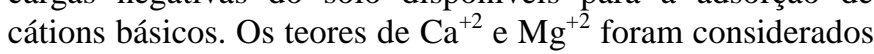
altos tanto na UEA agroecológica como na UEA convencional (EMBRAPA, 2006). Os resultados de pH nas UEA agroecológica $(7,79)$ e UEA convencional $(7,09)$ foram 
classificados como moderadamente alcalino e praticamente neutro, respectivamente (EMBRAPA, 2006).

Nas UEAs agroecológica e convencional os valores de soma de bases (SB) foram elevados, assim como os de saturação de bases (V), considerados eutróficos, ou seja, V maior que 50\% (EMBRAPA, 2006). Este comportamento está associado aos teores de MOS, e a predominância de cátions básicos. Estes resultados foram similares aos encontrados por Lira et al. (2012) em estudos da avalição da qualidade química de solos na Chapada do Apodi-RN. Tem-se que a V é um excelente indicador de avalição da fertilidade do solo, por reunir em termos estequiométricos macronutrientes $\left(\mathrm{Ca}^{2+}\right.$, $\mathrm{Mg}^{2+}$ e $\mathrm{K}^{+}$) essenciais para o crescimento e desenvolvimento vegetal. A CTC apresentou valor alto tanto na UEA agroecológica $\left(15,59 \mathrm{cmol}_{\mathrm{c}} \quad \mathrm{Kg}^{-1}\right)$ quanto na UEA convencional $\left(15,06 \mathrm{cmol}_{\mathrm{c}} \mathrm{Kg}^{-1}\right)$ (EMBRAPA, 2006) (Tabela 3).

Os valores de $\mathrm{P}$ assimilável nas UEA agroecológica $\left(111,23 \mathrm{mg} \mathrm{kg}^{-1}\right)$ e UEA convencional $\left(160 \mathrm{mg} \mathrm{kg}^{-1}\right)$ foram considerados altos (EMBRAPA, 2006). Estes valores de P foram bem superiores aos encontrados por Lira et al. (2012) em áreas com manejo da caatinga na Chapada do Apodi/RN $\left(13,76 \mathrm{mg} \mathrm{kg-}{ }^{1}\right)$ sem atividade agrícola. Provavelmente a elevação dos teores de $\mathrm{P}$ na UEA agroecológica está relacionada com a aplicação continuada de resíduos orgânicos e na UEA convencional com esterco de gado e fertilizantes mineralizados. Segundo Brady e Weil (2013), a dinâmica de $\mathrm{P}$ no solo tem ligação direta com a MOS na regulação deste nutriente fundamental para a fertilidade do solo e as plantas. Lourente et al. (2011) observaram correlação significativa ( $\mathrm{p}$ $\leq 0,01)$ e positiva $(\mathrm{r}=0,41)$ do $\mathrm{P}$ com o MOS em tratamentos com sistemas conservacionistas, evidenciando que há comportamento similar de elevação tanto para o $\mathrm{P}$ quanto para a MOS. Outra hipótese é da superestimação de $\mathrm{P}$ pelo método adotado, resultado de uma extração indevida de $\mathrm{P}$ não-lábil ligado ao $\mathrm{Ca}^{+2}(\mathrm{P}-\mathrm{Ca})$, comum em solos jovens com
pH elevados e altos níveis de $\mathrm{Ca}^{+2}$ trocáveis (NOVAIS; SMYTH, 1999).

A UEA agroecológica submetida ao manejo agroecológico apresentou IQS de 0,80 na camada estudada (Tabela 4), foi classificado como ótimo, mediante o enquadramento proposto por Freitas et al. (2012): IQS < 0,50 (ruim); IQS entre 0,50 a 0,70 (médio); IQS $\geq 0,71$ (ótimo). $\mathrm{O}$ IQS de 0,80 é superior aos valores obtidos por Freitas et al. (2012) em áreas de manejo com eucalipto em bases conservacionistas que oscilaram de 0,72 a 0,74 . Monteiro (2012) encontrou valor de IQS de 0,62 quando no cultivo de mandioca em consórcio com feijão de porco (Canavalia ensiformis) no Recôncavo da Bahia.

$\mathrm{Na}$ UEA agroecológica, a função que mais contribui para o índice de qualidade do solo foi a de SCN com 35,58\%, seguida por CR $(33,12 \%)$, RD $(21,55 \%)$ e CAA $(9,75 \%)$ (Tabela 4). Na SCN, a CTC foi a que mais elevou o índice com 31,30\% seguida da MOS (29,51\%), V (20,48\%), P assimilável $(10,50 \%)$ e $\mathrm{pH}(8,20 \%)$. Na CAA, os indicadores $\mathrm{UVcc} / \mathrm{PT}$ e $\mathrm{AD} / \mathrm{PT}$ apresentaram menores contribuições $0,07 \%$ e 3,61\%, respectivamente (Tabela 4). Estes baixos valores estão provavelmente associados aos quantitativos de areia presentes na UEA agroecológica (Tabela 2), que fizeram aproximar do limite da classe textural franco-arenosa à areia franca, que apresenta por sua vez baixa capacidade de retenção de água no solo. A continuidade de aportes de resíduos orgânicos no solo pode favorecer para elevar os valores dos indicadores físicos como UVcc/PT e AD/PT. Na função CR, a MOS foi que mais contribuiu $(31,70 \%)$ para o IQS, seguida da RP $(24,71 \%)$, PT $(22,45 \%)$ e Ds $(21,14 \%)$ (Tabela 4). Na RD, a MOS também ocupou posição de destaque $(64,98 \%)$ na elevação do IQS. As elevadas contribuições da MOS em todas as funções do solo é elemento sinalizador que a manutenção de resíduos orgânicos é fundamental para a QS, contribuindo para equilibrar as funções do solo na composição do IQS.

Tabela 4. Índice de qualidade do solo (IQS) na unidade experimental agrícola agroecológica $(0-20 \mathrm{~cm})$.

\begin{tabular}{|c|c|c|c|c|c|c|c|c|c|c|c|}
\hline \multirow{2}{*}{$\begin{array}{l}\text { Função } \\
\text { Principal }\end{array}$} & \multirow{2}{*}{$\begin{array}{l}\text { Ponderador } \\
\text { (A) }\end{array}$} & \multirow{2}{*}{$\mathrm{IQ}^{(1)}$} & \multirow{2}{*}{$\begin{array}{l}\text { Ponderador } \\
\text { (B) }\end{array}$} & \multirow{2}{*}{$\mathrm{VM}^{(2)}$} & \multirow{2}{*}{$\begin{array}{c}\text { Escores } \\
\text { (C) }\end{array}$} & \multicolumn{2}{|c|}{ (B) $\mathrm{x}(\mathrm{C})$} & \multirow{2}{*}{$\begin{array}{l}\text { Soma } \\
\begin{array}{l}\text { (B) } \mathrm{x}(\mathrm{C}) \\
= \\
\text { (D) }\end{array}\end{array}$} & \multicolumn{2}{|c|}{ (A) $x(D)$} & \multirow{2}{*}{$\begin{array}{l}\text { Índice de } \\
\text { qualidade } \\
\text { do solo }\end{array}$} \\
\hline & & & & & & absoluto & $(\%)$ & & absoluto & $(\%)$ & \\
\hline \multirow{4}{*}{ (CR) } & \multirow{4}{*}{0,30} & Ds & 0,20 & 1,32 & 0,93626 & 0,18725234 & 21,14 & \multirow{4}{*}{0,88596} & \multirow{4}{*}{0,27} & \multirow{4}{*}{33,12} & \multirow{15}{*}{0,80} \\
\hline & & $\mathrm{Rp}$ & 0,30 & 1,41 & 0,72976 & 0,218928 & 24,71 & & & & \\
\hline & & PT & 0,20 & 50,02 & 0,99452 & 0,19890335 & 22,45 & & & & \\
\hline & & MOS & 0,30 & 2,44 & 0,93626 & 0,28087851 & 31,70 & & & & \\
\hline \multirow{4}{*}{ CAA } & \multirow{4}{*}{0,20} & Ds & 0,20 & 1,32 & 0,94717 & 0,18943394 & 48,44 & & & & \\
\hline & & MOS & 0,20 & 2,44 & 0,93626 & 0,18725234 & 47,88 & 030108 & & 07472 & \\
\hline & & Uvcc/PT & 0,30 & 0,22 & 0,00086 & 0,00025677 & 0,07 & 0,39108 & 0,08 &, 7472 & \\
\hline & & $\mathrm{AD} / \mathrm{PT}$ & 0,30 & 0,11 & 0,04711 & 0,014132 & 3,61 & & & & \\
\hline \multirow{5}{*}{$\mathrm{SCN}$} & \multirow{5}{*}{0,30} & $\mathrm{pH}$ & 0,10 & 7,79 & 0,78089 & 0,07808945 & 8,20 & \multirow{5}{*}{0,95175} & \multirow{5}{*}{0,29} & \multirow{5}{*}{35,58} & \\
\hline & & CTC & 0,30 & 15,59 & 0,99300 & 0,29790 & 31,30 & & & & \\
\hline & & V & 0,20 & 85,62 & 0,97460 & 0,19492096 & 20,48 & & & & \\
\hline & & $P$ & 0,10 & 111,23 & 0,99960 & 0,09996 & 10,50 & & & & \\
\hline & & MOS & 0,30 & 2,44 & 0,93626 & 0,28087851 & 29,51 & & & & \\
\hline \multirow{2}{*}{$\mathrm{RD}$} & \multirow{2}{*}{0,20} & GF & 0,4 & 54,73 & 0,75694 & 0,30277579 & 35,02 & \multirow{2}{*}{0,86453} & \multirow{2}{*}{0,17} & \multirow{2}{*}{21,55} & \\
\hline & & MOS & 0,6 & 2,44 & 0,93626 & 0,56175702 & 64,98 & & & & \\
\hline
\end{tabular}

${ }^{(1)}$ Indicador de qualidade. ${ }^{(2)}$ Valor médio observado. ${ }^{(3)}$ Escores padronizados (normalizados). ${ }^{(4)}$ Índice de qualidade do solo. CR: Crescimento radicular. CAA: Condução e armazenamento de água. SCN: Suprir e ciclar nutrientes. RD: Resistir à degradação.

Na UEA convencional, as funções como SCN $(50,30 \%)$, CR $(22,63 \%)$, CAA $(20,89 \%)$ e RD $(6,18 \%)$ apresentaram as suas contribuições em ordem decrescente para o IQS de 0,45 , considerado como ruim (FREITAS et al., 2012) (Tabela 5). A
RD foi a função do solo que mais influenciou para a diminuição do IQS, onde o GF foi responsável por apenas $0,74 \%$, enquanto a MOS contribuiu com 99,26\% (Tabela 5). Na SCN, a CTC foi responsável por $39,36 \%$ para os $50,30 \%$ 
da referida função. Na CAA, a AD/PT $(63,77 \%)$ foi que mais contribuiu para elevar a função do solo na condução e armazenamento de água, enquanto a MOS só contribuiu com 9,78\%. A AD/PT está associada à proporção de água disponível do solo. Infere-se que menor PT e maior Ds influenciaram na distribuição de poros do solo, fazendo aumentar a microporosidade (retenção de água) e diminuir a macroporosidade (percolação de água).

É fundamental a adoção de práticas agrícolas que elevem a reposição de resíduos orgânicos na UEA convencional, contribuindo para a cobertura do solo. Deve-se ainda diminuir o revolvimento do solo. O aumento da MOS proporciona a melhor estruturação do solo, diminuição da ADA e aumento do GF. Com a aplicação do IQS, percebe-se que a QS não se quantifica apenas com indicadores isolados, mas, sobretudo, em conjunto com os demais, onde todas as funções do solo com seus indicadores associados têm importância na QS. Um dos grandes problemas dos solos do semiárido é a susceptibilidade a processos de erosão. Neste contexto, o IQS foi uma ferramenta fundamental para orientar o uso e manejo do solo na UEA convencional, que além de manter a SCN, contribui para elevar a RD ao longo do tempo.

Tabela 5. Índice de qualidade do solo (IQS) na unidade experimental agrícola convencional $(0-20 \mathrm{~cm})$.

\begin{tabular}{|c|c|c|c|c|c|c|c|c|c|c|c|}
\hline \multirow{2}{*}{$\begin{array}{l}\text { Função } \\
\text { Principal }\end{array}$} & \multirow{2}{*}{$\begin{array}{l}\text { Ponderador } \\
\text { (A) }\end{array}$} & \multirow{2}{*}{$\mathrm{IQ}^{(1)}$} & \multirow{2}{*}{$\begin{array}{l}\text { Ponderador } \\
\text { (B) }\end{array}$} & \multirow{2}{*}{$\mathrm{VM}^{(2)}$} & \multirow{2}{*}{$\begin{array}{l}\text { Escores } \\
{ }^{(3)}(\mathrm{C})\end{array}$} & \multicolumn{2}{|c|}{ (B) $\mathrm{x}(\mathrm{C})$} & \multirow{2}{*}{$\begin{array}{c}\text { Soma } \\
\begin{array}{c}\text { (B) } \mathrm{x}(\mathrm{C}) \\
= \\
\text { (D) }\end{array}\end{array}$} & \multicolumn{2}{|c|}{ (A) $\mathrm{x}(\mathrm{D})$} & \multirow{2}{*}{$\begin{array}{l}\text { Índice de } \\
\text { qualidade } \\
\text { do solo }\end{array}$} \\
\hline & & & & & & absoluto & $(\%)$ & & absoluto & $(\%)$ & \\
\hline \multirow{4}{*}{$\mathrm{CR}$} & \multirow{4}{*}{0,30} & Ds & 0,20 & 1,60 & 0,47385 & 0,094770509 & 27,91 & \multirow{4}{*}{0,33959} & \multirow{4}{*}{0,10} & \multirow{4}{*}{22,63} & \multirow{15}{*}{0,45} \\
\hline & & $\mathrm{Rp}$ & 0,30 & 2,21 & 0,41331 & 0,123991539 & 36,51 & & & & \\
\hline & & PT & 0,20 & 37,95 & 0,25911 & 0,051821109 & 15,26 & & & & \\
\hline & & MOS & 0,30 & 1,22 & 0,23003 & 0,069008656 & 20,32 & & & & \\
\hline \multirow{4}{*}{ CAA } & \multirow{4}{*}{0,20} & Ds & 0,20 & 1,60 & 0,47385 & 0,094770509 & 20,15 & & & & \\
\hline & & MOS & 0,20 & 1,22 & 0,23003 & 0,046005771 & 9,78 & 047028 & 009 & 208908 & \\
\hline & & Uvcc/PT & 0,30 & 0,44 & 0,09874 & 0,029621289 & 6,30 & $0,4 / 0<8$ & 0,09 & 20,8908 & \\
\hline & & $\mathrm{AD} / \mathrm{PT}$ & 0,30 & 0,22 & 0,99960 & 0,299879999 & 63,77 & & & & \\
\hline \multirow{5}{*}{$\mathrm{SCN}$} & \multirow{5}{*}{0,30} & $\mathrm{pH}$ & 0,10 & 7,09 & 0,94830 & 0,094830464 & 12,56 & \multirow{5}{*}{0,75494} & \multirow{5}{*}{0,23} & \multirow{5}{*}{50,30} & \\
\hline & & CTC & 0,30 & 15,06 & 0,99048 & 0,29714 & 39,36 & & & & \\
\hline & & V & 0,20 & 84,00 & 0,96999 & 0,193998951 & 25,70 & & & & \\
\hline & & $\mathrm{P}$ & 0,10 & 160,55 & 0,99960 & 0,09996 & 13,24 & & & & \\
\hline & & MOS & 0,30 & 1,22 & 0,23003 & 0,069008656 & 9,14 & & & & \\
\hline \multirow{2}{*}{$\mathrm{RD}$} & \multirow{2}{*}{0,20} & GF & 0,4 & 25,99 & 0,00256 & 0,001022624 & 0,74 & \multirow[b]{2}{*}{0,13904} & \multirow[b]{2}{*}{0,03} & \multirow[b]{2}{*}{6,18} & \\
\hline & & MOS & 0,6 & 1,22 & 0,23003 & 0,138017313 & 99,26 & & & & \\
\hline
\end{tabular}

(1) Indicador de qualidade. ${ }^{(2)}$ Valor médio observado. ${ }^{(3)}$ Escores padronizados (normalizados). ${ }^{(4)}$ Índice de qualidade do solo. CR: Crescimento radicular. CAA: Condução e armazenamento de água. SCN: Suprir e ciclar nutrientes. RD: Resistir à degradação.

\section{CONCLUSÕES}

$O$ Índice de qualidade do solo (IQS) na UEA agroecológica foi melhor classificado que na UEA convencional. Na UEA convencional, há necessidade de práticas agrícolas como o aporte de resíduos orgânicos que elevem a RD ao longo do tempo, como a melhoria do GF e diminuição da ADA, pois foi a função do solo que mais contribuiu para reduzir o IQS.

Os IQS das UEAs refletiram o desempenho integrado nas áreas estudadas. É uma ferramenta útil que poderá ser utilizada nas tomadas de decisão sobre o uso e manejo do solo que elevem o IQS, visando à sustentabilidade ambiental dos sistemas agrícolas irrigados em convivência com semiárido.

\section{AGRADECIMENTOS}

Ao Projeto Dom Helder Câmara (PDHC) do Ministério do Desenvolvimento Agrário (MDA), em colaboração com o Fundo Internacional para o Desenvolvimento da Agricultura (FIDA) e o Fundo Global para o Meio Ambiente (GEF) pelo estudo ter sido realizado em área de sua atuação.

\section{REFERÊNCIAS}

ALVARENGA, C. C.; MELlO, C. R.; MELlO, J. M.; SILVA, A. M.; CURI, N. Índice de Qualidade do Solo associado à recarga de água subterrânea (IQSRA) na Bacia
Hidrográfica do Alto Rio Grande, MG. Revista Brasileira de Ciência do Solo, v.36, p.1608-1619, 2012.

ARAÚJO FILHO, J. A. de. Manejo pastoril sustentável da caatinga. Recife, PE: Projeto Dom Helder Camara, 2013. 200p.

AZEVEDO, A. C.; DALMOLIN, R. S. D. Solos e ambiente: Uma introdução. 2 ed. Santa Maria, Pallotti, 2006. 100 p.

BHARDWAJ, A. K.; JASROTIA, P.; HAMILTONA, S. K.; ROBERTSON, G. P. Ecological management of intensively cropped agro-ecosystems improves soil quality with sustained productivity. Agriculture. Ecosystems \& Environment, v.140, p.419-429, 2011.

BLAINSKI, E.; TORMENA, C. A.; FIDALSKI, J.; GUIMARÃES, R. M. L. Quantificação da degradação física do solo por meio da curva de resistência do solo à penetração. Revista Brasileira de Ciência do Solo, v.32, p.975-983, 2008.

BRADY, N. C.; WEIL, R. R. Elementos da natureza e propriedades dos solos. Tradução técnica: Igo Fernando Lepsch. Dados eletrônicos. Porto Alegre: Bookman, 2013. $716 \mathrm{p}$.

BRAGA, J. M.; DEFELIPO, B. V. Determinação espectrofotométrica de fósforo em extratos de solo e material vegetal. Revista Ceres, v. 21, p.73-85, 1974. 
CAMARGO, O. A.; ALlEONI, L. R. F. Compactação do solo e o desenvolvimento das plantas. Piracicaba: ESALQ/USP, 1997. 132p.

CÂNDIDO, B. M. Erosão hídrica e qualidade do solo em sistemas florestais no leste do Mato Grosso do Sul. 2014. 93f. Dissertação (Mestrado em Ciência do Solo) - Universidade Federal de Lavras, Lavras. 2014.

CHEN, Y. D.; WANG, H. Y.; ZHOU, J. M.; XING, L.; ZHU, B. S.; ZHAO, Y. C.; CHEN, X. Q. Minimum data set for assessing soil quality in farmland of Northeast China. Pedosphere 23(5): 564-576, 2013. ISSN 1002-0160/CN $32-$ 1315/P. Soil Science Society of China. p. 564-576. DOI: https://doi.org/10.1016/S1002-0160(13)60050-8.

CORRÊA, R. M.; FREIRE, M. B. G. S.; FERREIRA, R. L. C.; FREIRE, F. J.; PESSOA, L. G. M.; MIRANDA, M. A.; MELO, D. V. M. Atributos químicos de solos sob diferentes usos em perímetro irrigado no semiárido de Pernambuco. Revista Brasileira de Ciência do Solo. Viçosa, v.33, n.2, 2009.

DANTAS, J. D.; OLIVEIRA, T. S.; MENDONÇA, E. S.; ASSIS, C. P. Qualidade de solo sob diferentes usos e manejos no Perímetro Irrigado Jaguaribe/Apodi, CE. Revista Brasileira de Engenharia Agrícola e Ambiental, Campina Grande, PB, v. 16, n. 1, p. 18-26, 2012.

EMBRAPA. EMPRESA BRASILEIRA DE PESQUISA AGROPECUÁRIA. Sistema brasileiro de classificação de solos. Rio de Janeiro: Centro Nacional de Pesquisa de Solos, 2006. 306p.

EMBRAPA. EMPRESA BRASILEIRA DE PESQUISA AGROPECUÁRIA. Manual de análises químicas de solos, plantas e fertilizantes - 2. ed. rev. ampl. - Brasília, DF : Embrapa Informação Tecnológica, 2009. 627 p.

FREITAS, D. A. F.; SILVA, M. L. N.; CARDOSO, E. L.; CURI, N. Índices de qualidade do solo sob diferentes sistemas de uso e manejo florestal e cerrado nativo adjacente. Revista Ciência Agronômica, v. 43, n. 3, p. 417-428, 2012.

KARLEN, D. L.; STOTT, D. E. A framework for evaluating physical and chemical indicators of soil quality. In: DORAN, J. W.; COLEMAN, D. C.; BEZDICEK, D. F.; STEWART, B. A., eds. Defining soil quality for a sustainable environment. Madison, SSSA, p.53-72, 1994. (Special, 35).

KIEHL, E. J. Manual de edafologia: Relações solo-planta. São Paulo, Agronômica Ceres, 1979. 262 p.

LIRA, R. B. de.; DIAS, N. S.; ALVES, S. M. C.; BRITO, R. F. de.; SOUSA NETO, O. N. de. Efeitos do sistemas de cultivo e manejo da caatinga através da análise dos indicadores químicos de qualidade do solo na produção agrícola em Apodi, RN. Revista Caatinga, v. 25, n. 3, p. 1824,2012

LOURENTE, E. R. P.; MERCANTE, F. M.; ALOVISI, A. M. T.; GOMES, C. F.; GASPARINI, A. S.; NUNES, C. M.
Atributos microbiológicos, químicos e físicos de solo sob diferentes sistemas de manejo e condições de Cerrado Pesquisa Agropecuária Tropical, v. 41, n. 1, p. 20-28, 2011

MCBRATNEY, A.; FIELD, D. J.; KOCH, A. The dimensions of soil security. Geoderma, v. 213, 203-213, 2013. DOI: https://doi.org/10.1016/j.geoderma.2013.08.013

MELO FILHO, J. F.; CARVALHO, L. L.; SILVEIRA, D. C.; SACRAMENTO, J. A. A. S.; SILVEIRA, E. C. P. Índice de qualidade em um Latossolo Amarelo Coeso cultivado com citros. Revista Brasileira de Fruticultura, v. 31, n. 4, p. 1168 1177, 2009.

MENDONÇA, E. S.; MATOS, E. S. Matéria orgânica do solo: Métodos de análises. Viçosa, MG, Universidade Federal de Viçosa, 2005, 107p.

MIRANDA, M. C. Capacidade produtiva e qualidade de sítios cultivados com eucalipto em argissolos de tabuleiro no Litoral Norte da Bahia. 2012. 121f. Dissertação (Mestrado em Solos e Qualidade de Ecossistemas) - Universidade Federal do Recôncavo da Bahia, Cruz das Almas. 2012.

MONTEIRO, J. F. Avaliação da qualidade do solo em diferentes sistemas de uso e manejo agrícola em ambiente tropical. 2012. 66f. Dissertação (Mestrado em Solos e Qualidade de Ecossistemas) - Universidade Federal do Recôncavo da Bahia, Cruz das Almas. 2012.

MOTA, J. C. A.; FREIRE, A. G.; ASSIS JÚNIOR, R. N. Qualidade física de um Cambissolo sob sistemas de manejo. Revista Brasileira de Ciência do Solo, v.37, p.1196 - 1206, 2013.

NOVAIS, R. F.; SMYTH, T. J. Fósforo em solo e planta em condições tropicais. Viçosa, MG, Universidade Federal de Viçosa, 1999. 399p.

OBADE, V. P.; LAL, R. Soil quality evaluation under different land management practices. Environ. Earth Sci., doi: 10.1007/s12665-014-3353-z, 2014.

RICHARDS, L. A. Diagnosis and improvement of saline and alkali soils. Washington, United States Department of Agriculture, 1954. 160p. (Agriculture Handbook, 60).

ROSOLEM, C. A.; FERNANDEZ, E. M.; ANDREOTTI, M.; CRUSCIOL, C. A. C. Crescimento radicular de plântulas de milho afetado pela resistência do solo à penetração. Pesquisa Agropecuária Brasileira, v.34, p.821-828, 1999.

SANTIAGO, F. S. Avaliação da qualidade do solo em sistemas de cultivo irrigado agroecológico e convencional no semiárido do Rio Grande do Norte. 2015. 175f. Tese (Doutorado em Engenharia Agrícola) - Universidade Federal Rural de Pernambuco, Recife. 2015.

SILVA, M. A.; FREITAS, D. A. F.; SILVA, M. L. N.; OLIVEIRA, A. H.; LIMA, G. C.; CURI, N. Sistema de informações geográficas no planejamento de uso do solo. Revista Brasileira de Ciências Agrárias, v.8, n.2, p.316-323, 2013. 
SOUZA, A. L. V. Avaliação da qualidade de um Latossolo Amarelo Coeso argissólico dos Tabuleiros Costeiros, sob floresta natural. 2005. 114f. Dissertação (Mestrado em Ciências Agrárias) - Universidade Federal da Bahia, Cruz das Almas. 2005.

STOLF, R. Teoria e teste experimental de fórmulas detransformação dos dados de penetrômetro de impacto em resistência do solo. Revista Brasileira de Ciência do Solo. Campinas, v.15, p.249-252, 1991.

WALKLEY, A.; BLACK, I. A. An Examination of Degtjareff Method for Determining Soil Organic Matter and a Proposed Modification of the Chromic Acid Titration Method. Soil Science, v.37, p.29-37, 1934.

WYMORE, A. W. Model-based systems engineering: an introduction to the mathematical theory of discrete systems and to the tricotyledon theory of system design. Boca Raton, Florida, USA: CRC Press, 1993.

YAO, R. J.; YANG, J. G.; ZHANG, T. J.; GAO, P.; YU, S. P.; WANG, X. P. Short-term effect of cultivation and crop rotation systems on soil quality indicators in a coastal newly reclaimed farming area. Journal Soils Sediments, v.13, p.1335-1350, 2013. 